\title{
LARGE APERTURE MAGNETS FOR A FUTURE HIGH POWER PROTON SYNCHROTRON*
}

\author{
J.-F. Ostiguy and F. Mills, FNAL, Batavia, IL 60510, USA
}

\begin{abstract}
A high intensity, high power proton synchrotron is currently under consideration at Fermilab. The machine known as the Proton Driver - would accelerate $3 \times 10^{13}$ protons from $400 \mathrm{MeV}$ to $12 \mathrm{GeV}$ (stage I) or $16 \mathrm{GeV}$ (stage II) and ultimately deliver in excess of $1 \mathrm{MW}$ of beam power. To minimize losses and insure beam stability, the space charge-induced tune shift must be kept well below 0.5 . This is accomplished by spreading out bunches both longitudinally and transversely. While the former strategy favors high voltage low frequency RF, the latter leads to magnets with unconventionally large apertures. This requirement, combined with a $1.5 \mathrm{~T}$ bending field and rapid cycling operation results in a number of serious but not insurmountable challenges. In this paper, we discuss the design of the Proton Driver magnets and the rationale behind it.
\end{abstract}

\section{INTRODUCTION}

The Fermilab Proton Source chain was constructed in the early 1970's. Since then, the Booster synchrotron has remained essentially the same machine. The advent of the new Main Injector synchrotron and its ability to simultaneously support the Tevatron collider and $120 \mathrm{GeV}$ fixed target programs, has stimulated the demand for protons. Two experiments are now in construction: the Neutrinos at the Main Injector (NUMI) project and MiniBoone (Booster Neutrinos). In that context, a study was undertaken to investigate the technical feasibility and the cost of a new high intensity proton synchrotron. The machine would ultimately be capable of delivering $3 \times 10^{13}$ protons/pulse at $15 \mathrm{~Hz}$. The rationale for the design choices is presented in details in reference [1] and is the object of other publications presented at this conference. This paper focuses on the magnet system and discusses design considerations, challenges and technical solutions.

\section{LATTICE}

The existing FNAL $8 \mathrm{GeV}$ Booster has a simple FODO-like lattice based on combined function magnets. Combined function magnets offer several advantages: they saves space, reduce cost and naturally ensure excellent dipole/quadrupole strength tracking. In contrast, the proposed Proton Driver synchrotron is a transition-less $\left(\gamma_{t}^{2}<0\right)$ machine with a lattice based on flexible momentum compaction factor (FMC) cells whose realization implies separated functions magnets. While this makes dipole/quadrupole tracking an issue, it can be addressed

\footnotetext{
* Work supported Universities Research Association Inc., under contract DE-AC02-76CH0-3000 with the U.S. Department of Energy.
}

with a dynamic correction system. Higher bending fields can also be reached since conventional dipoles tend to saturate more gently than combined function magnets whose geometry introduces higher order harmonics that are difficult to compensate for.

\section{DIPOLE MAGNETS}

A cross-section of the Proton Driver dipole magnet is shown in Figure 1. This is a conventional H-magnet with poles profiled so as to prevent early saturation in the edges regions [2]. The magnet is completely enclosed in an external vacuum skin, eliminating the need for a beam vacuum chamber. Thin metallic strips along the pole surface allow image currents circulate and minimize beam impedance. Each pole is driven by two identical 12-turn coils connected in parallel.

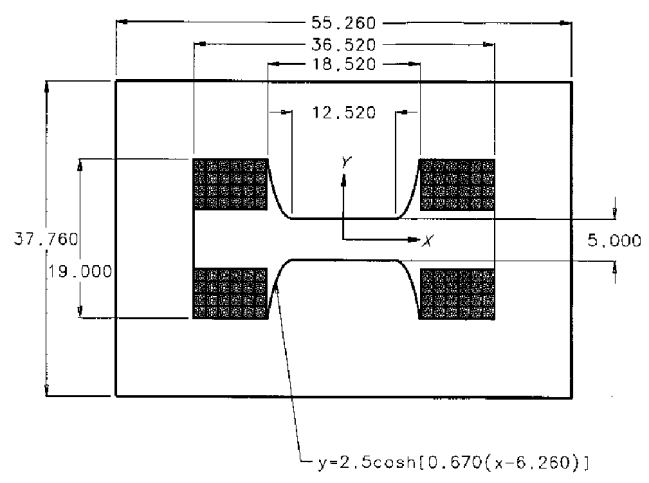

Figure 1: Proton Driver Dipole Magnet Cross Section.

\subsection{Field Strength and Physical Aperture}

Since they ultimately determine magnet size and stored energy, field strength and physical aperture are primary costs drivers. Clearly, a higher field strength is desirable because it reduces the machine circumference; however, in a rapid cycling synchrotron where space charge induced tune shift is significant, it is necessary to provide excellent dipole/quadrupole strength tracking. The dipole field was set to $1.5 \mathrm{~T}$; although this is not a hard limit, the amount of dynamic correction required increases very rapidly beyond this value.

To minimize the space-charge induced tune shift, charge density is reduced by spreading out the bunch distribution longitudinally and/or transversely. While the former remedy implies low frequency RF, the latter implies large transverse physical aperture. The proton driver transverse bunch dimensions (at injection) are 5.5 in $\times 3.5$ in. Additional 
clearance is provided to keep losses a level compatible with safety requirements. With a physical aperture $12.5 \times 5 \mathrm{in}^{2}$, injection losses are expected to be $<10 \%$.

\subsection{Field Quality}

Field homogeneity is an important issue, and while ultimately extensive tracking is necessary to objectively determine how good it needs to be, for costing purposes, a reasonable assumption based on accumulated experience is that it should be better than $5 \times 10^{-4}$ over the entire beam and better than $10^{-3}$ over the entire "good field region". For the proton driver, this "good field region" is 5 in $\times 9$ in. For a conventional iron dipole magnet, to achieve a specified field homogeneity at the edge of the good field region, it is necessary to extend the physical horizontal aperture by an amount referred to as the pole overhang $d$. For given field homogeneity and gap size $g, d$ can be determined using a formula due to K. Halbach [3] which estimates the field homogeneity achievable with a suitably shimmed profile. As illustrated in Figure 2, Halbach's formula shows field homogeneity in the good field region can be improved, if need be, by roughly an order of magnitude each time $d$ is increased by $0.15 \mathrm{~g}$.

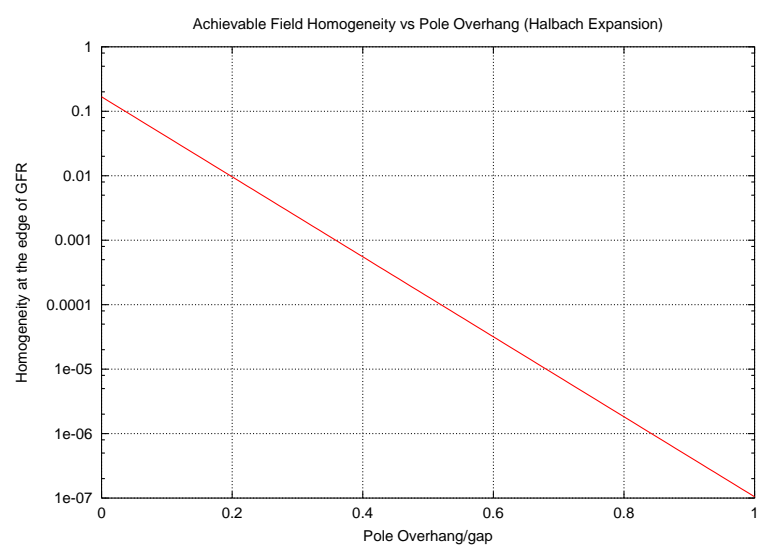

Figure 2: Achievable Field Homogeneity, from Reference[3].

\subsection{Stored Energy}

An important consequence of large physical aperture is large stored energy. Because magnetic energy must be cycled in an out of the ring at $15 \mathrm{~Hz}$, a resonant power supply is the most economically viable alternative and its cost is expected to scale linearly with the stored energy. In that context, an interesting observation is that for a conventional $\mathrm{H}$ magnet, a surprisingly large fraction $(\simeq 40 \%)$ of the stored field energy is located in the fringing regions. It is plausible that a proper combination of coil positioning and pole shaping might reduce this fraction. This avenue has not been pursued.

\subsection{High Voltage}

Large stored energy and rapid cycling frequency result in magnets that have to sustain significantly higher voltage-toground than what Fermilab has operational experience with $(5 \mathrm{kV})$. The voltage can be reduced by reducing the number of turns in the coils; however, at fixed field, the total current linked by each pole must remain constant and reducing the number of turns increases the current carried by each conductor. Since higher current implies higher resistive losses, a compromise must be reached. For the Proton Driver dipoles, the voltage to ground reaches a maximum of $5 \mathrm{kV}$ (16 GeV operation) and conductors carry a maximum current of $6700 \mathrm{~A}$. This was accomplished by splitting each coil into two parts connected in parallel.

High voltage insulation is obviously a concern for reliable long term operation. The design and optimization of the insulation, especially in the end regions is likely to be challenging. Fortunately, experience with ISIS at RAL provides concrete evidence that reliable high voltage magnet operation is possible.

\subsection{AC Losses}

Rapid cycling rate impacts steel losses. To keep them under control, both the dipole and the quadrupole magnets are constructed with 0.014 in M17 Si-Fe laminations. This material is similar to the material used in power transformers.

Another consequence is that the effect of eddy currents in the coils cannot be overlooked. When eddy currents are treated as a perturbation, it can be shown that the power dissipated in a circular conductor subjected to a uniform external transverse field $B$ is

$$
P \simeq \frac{\pi}{4} \sigma r^{4}(\dot{B})^{2}
$$

where $r$ is the conductor radius, $(\dot{B})$ is the time derivative of the field $B$ and $\sigma$ is the electrical conductivity. While this result is not applicable to situations where the presence of eddy currents significantly affect the current distribution in the conductor cross-section, it nevertheless illustrates that the dissipated power increase very rapidly with the conductor average radius. For reference, the $8 \mathrm{GeV}$ FNAL Booster magnets, which also operate at $15 \mathrm{~Hz}$ have coils made out of solid water-cooled conductor 0.45 in $\times 0.45$ in crosssection. Self-consistent calculations using a standard finite element code show that in that case, the ratio

$$
\mathcal{R}=\frac{R_{\mathrm{AC}}}{R_{\mathrm{DC}}} \simeq 2
$$

For the sake of comparison, if the same type of conductor were to be used for the Proton Driver dipole, $\mathcal{R} \simeq 8$, which clearly, is not acceptable. Now, the Booster magnet conductor is close to the smallest practical size for a water cooled conductor. Assuming even smaller cross-section conductor could be fabricated, a large number of turns would be required and complex mechanical connections and conductor 
transposition would be required to prevent the voltage from becoming unreasonably high.

Recently, the Japanese industry has developed a water cooled stranded conductor that eliminates most of the difficulties associated with eddy currents. As illustrated in Figure 3, this conductor is made out of electrically insulated $2 \mathrm{~mm}$ strands surrounding a solid water cooling pipe. The strands are transposed longitudinally to ensure uniform current density and can be either $\mathrm{Al}$ or $\mathrm{Cu}$. For $\mathrm{Al}$, interstrand insulation is provided by aluminum oxide. For $\mathrm{Cu}$, the strands are coated with an insulating material such as polyimide.

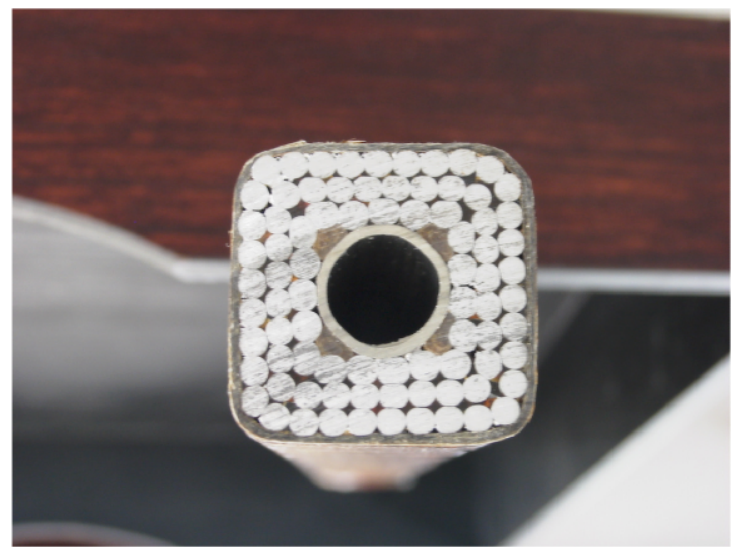

Figure 3: Water-cooled Stranded Conductor Cross-section.

\subsection{Tracking}

A question which arises naturally is how good should the quadrupole/dipole tracking be. With respect to a particle at the nominal (reference) momentum, a particle of momentum $p+\Delta p$ will experience a tune shift

$$
\Delta \nu=\xi_{\text {uncorrected }}\left[\frac{\Delta G}{G}-\frac{\Delta B}{B}\right]+\xi_{\text {corrected }} \frac{\Delta p}{p}
$$

where $\xi$ is the chromaticity. We note that a quadrupole tracking error is equivalent to a momentum error, with the important difference that it is affected by the uncorrected chromaticity. This results from the fact that by design, the bend field always tracks the particle momentum. Since quadrupole errors do not result in closed orbit distortions, chromaticity correction has no effect.

Quadrupole tracking errors induce a coherent tune shift which effectively displaces the entire beam tune footprint. How much tune shift is tolerable ultimately depends on the extent and location of the beam footprint in tune space. Nevertheless, based on the ISIS experience, the Proton Driver design assumes $\delta \nu<0.01$ which leads roughly to

$$
\left[\frac{\Delta G}{G}-\frac{\Delta B}{B}\right]<0.001
$$

since the uncorrected chromaticity is $\sim 10$. In order to achieve this level of tracking, the dipole and share a common current bus. The residual error is handled by a dynamic correction system.

\section{QUADRUPOLE MAGNETS}

The Proton Driver quadrupole cross-section is shown in Figure 4. The quadrupole has a four-fold symmetric crosssection. This provides natural higher harmonic suppression at the expense of overall size, since the horizontal beam size sets the aperture radius. The quadrupole gradient is limited by saturation. For a large aperture quadrupole, the achievable interior pole tip field decreases with aperture size. The pole tip field of the Proton Driver quadrupole is approximately $0.75 \mathrm{~T}$ and $1.5 \mathrm{~T}$ at the edge of the horizontal aperture. The gradient is $8.74 \mathrm{~T} / \mathrm{m}$.

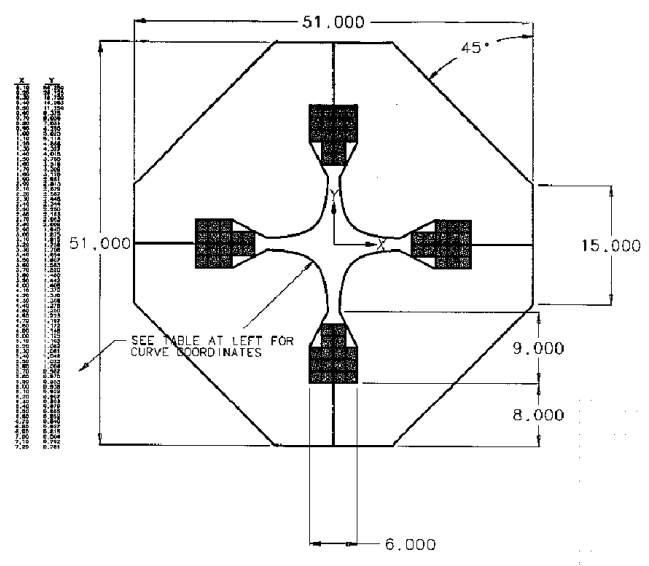

Figure 4: Proton Driver Quadrupole Cross-Section.

\section{OTHER MAGNETS}

The Proton Driver sextupole correction magnets have a six-fold symmetric cross-section and are independently powered. Just as for the quadrupole, symmetry provides natural higher harmonic suppression.

The vertical correctors are of a standard pole-less design. This design has the advantage of providing good field quality even under moderate saturation levels, at the expense of lower efficiency. High efficiency is not a major concern for small orbit correction magnets. There is very little free longitudinal space in the proton driver lattice. To save space, it is envisioned that horizontal correction could be integrated into the end region of the dipole magnets. This would requires auxiliary trim coils, as well as a scheme to decouple the trim power supply from the main power supply. However, the cost and complexity of such a system favor relaxing some lattice constraints in order to provide sufficient space for more conventional horizontal correctors.

\section{REFERENCES}

[1] “The Proton Driver Design Study", Fermilab Publication TM2136, December 2000.

[2] S.Snowdon, Fermilab Publication FN-184

[3] K. Halbach in "Handbook of Accelerator Physics and Engineering", A. Chao ed., World Scientific (1998) 